\title{
MINERALOGICAL AND CHEMICAL CHARACTERIZATION OF THE SOIL FROM BÂRZAVA RIVER AREA
}

\author{
DELIA PIRŞAN ${ }^{\mathrm{a}}$, ANAMARIA TOROK ${ }^{\mathrm{b}}$, CLAUDIU TĂNĂSELIA $^{\mathrm{b}}$, \\ PAULA PODEA ${ }^{\mathrm{c}}$
}

\begin{abstract}
Mineralogical and chemical characterization of soils is important in for the use of soils as agricultural land. The soil from downstream Bârzava River was characterized and existing geological features of this soil were presented and correlate with the size surfaces. $\mathrm{pH}$ measurements of soils samples collected from three different locations and from two different depths were performed. The soil samples were also analysed in order to assess the major and trace elements content. It is known that the $\mathrm{pH}$ and heavy metal concentrations are vital factors for plant growth and the obtained results could be use in future for elaboration of some soil bioremediation techniques.
\end{abstract}

Keywords: soil geological features, acidity control, metal concentration

\section{INTRODUCTION}

Acid soils occupy approximately $30 \%$ of the world's land area and restrain global agricultural production ${ }^{1}$. Numerous factors can contribute to soil acidification, such as large inputs of inorganic fertilizers, high rainfall, acid deposition and greenhouse gas ${ }^{2}$. As the concentration of $\mathrm{H}+$ in the soil increases, it can inhibit root growth $^{3}$, disrupt the functions of the plasma membrane $e^{4}$, cell wall ${ }^{5}$, or increase the $\mathrm{Al}^{3+}$ toxic levels ${ }^{6}$. Deficient levels of calcium $(\mathrm{Ca})$, magnesium $(\mathrm{Mg})$ and phosphorus $(\mathrm{P})$ are also frequent under low $\mathrm{pH}$ conditions.

a Technical University of Cluj-Napoca, Faculty of Materials and Environmental Engineering, Bd. Muncii Str., No. 103-105, RO-40064, Cluj-Napoca, Romania

b INCDO-INOE 2000 Research Institute for Analytical Instrumentation ICIA, 67 Donath Str., RO-400293 Cluj-Napoca, Romania

c Universitatea Babeş-Bolyai, Facultatea de Chimie şi Inginerie Chimică, Kogălniceanu Str., No. 1, RO-400084, Cluj-Napoca, Romania

*Corresponding author: delia_pirsan@yahoo.com 
Soil $\mathrm{pH}$ is widely accepted as a dominant factor that regulates soil nutrient bioavailability, vegetation community structure, plant primary productivity, and a range of soil processes including soil microbial community structure and activity?.

All the soil properties and the value of the soil pH can widely differ in reliance on soil type, topography, climate, vegetation, and anthropogenic activity, because all these factors influence the spatial variability of the observed soil types ${ }^{8}$. The value of soil $\mathrm{pH}$ is directly influenced by all five soilforming factors (parent rock, climatic conditions, organisms, topography, and time) and further the value of soil $\mathrm{pH}$ is dependent on the season influence, way of management, tested soil horizon, soil water contents, and time limit of sampling for analysis ${ }^{9}$. In literature there are only some few studies regarding the correlation between soil acidity and agricultural plant cultures cultivated in Romania. Some studies and researches at national level have pointed out that there are relations of interdependence between plant cultivation technologies, the environment, the level of economic development and the quality of life ${ }^{10,11,12,13 a, b, 14}$. Anthropogenic metals and metalloids in soils represent a potential risk for the environment. Accumulation of the heavy metals in agricultural soils and water resources poses a great threat for the living organism as well for the human health (due to high risk of their entry into food chain $)^{15}$. Prolonged consumption of contaminated vegetables and cereals can lead to the disruption of numerous biological and biochemical processes in the human body ${ }^{16}$.

Taking into account all these, our paper presents eco-pedological research in the area of the Bârzava River, Caraş-Severin County, Romania, from a soil chemistry perspective, aiming at establishing improvement measures. The focus has been on soils characterization and determination of acidity and metal concentrations of soils samples. The characterization of soils, from this area, is important for the future studies regarding soils bioremediation.

\section{RESULTS AND DISCUSSION}

The study focussed on the lands downstream Bârzava River, CaraşSeverin County, Romania. The main soil types identified in the studied area, where soils with specific features have developed in close relationship with the variety of geomorphologic factors determining the existence of diversified relief units, of geolitic and hydrologic factors, and of different anthropogenic factors. Six soil samples were collected from three different locations in 
Bârzava river area, two different samples (from 0-20 cm and 20-40 cm) from each location (Voiteni, Partoş, Banloc-Livezile) Samples were codified as A, B, C, D, E, F. (A soil sample from Banloc-Livezile pump station area (0-20 $\mathrm{cm})$; B. soil sample from Partoş rice plantation area $(0-20 \mathrm{~cm})$; C. soil sample from Voiteni Farm of USAMVBT $(0-20 \mathrm{~cm})$; D. soil sample from wheat plantation area from Voiteni Farm of USAMVBT $(20-40 \mathrm{~cm})$; E. soil sample from Banloc-Livezile pump station area $(20-40 \mathrm{~cm})$; $F$ soil sample from Partoş rice plantation area $(20-40 \mathrm{~cm})$ ). Samples were mineralogical and chemical characterized, acidity and metal concentrations were determined.

Bârzava River is a river of $166 \mathrm{~km}$ long in Caraş-Severin County situated in south-west part of Romania. Location coordinates are 45 $17^{\prime} 59^{\prime \prime} \mathrm{N}$ 203' 46 "'E.

In the low plain downstream Bârzava River, the soil type is gleic eutricambosoil, moderately gleized, moderately decarbonized (balticalcaric), medium sandy clay/medium clay, developed on medium fluvial carbonate materials.

Soil from downstream Bârzava River was characterized and main features of this soil were presented in Table 1.

The soil horizon or pedogenetic horizon is a layer approximately parallel to the soil surface, which has a number of properties resulting from the soil formation process, properties that differ from those of the above or underlying strains. The soil horizon is meant a component layer of the profile, characterized by the entire mass of the same properties (color, texture, structure, etc.). The Ao ocric horizon is a too bright or too bioaccumulative horizon in organic matter to be molluscic or shady, or which becomes massive and harsh during periods of drought. The $A B$ horizon is a transition horizon between $A$ and $B$, with horizon $A$ properties and rock fragments, which are at least $30 \%$ altered. The Bvg2 horizon is a cambic surface horizon (change-over, modification), also known as the horizon of alteration of the parental material in situ, g2 signifies the degree of gleizing-weakness. BCg2 horizon is a transition horizon between $B$ and $C$ horizon, with the characters of the supriacent $B$ horizon and the underlying $C$ being partially expressed, with a glearing degree (g2). Ckg3 horizon is underlying material, having a moderate (g3) degree of accumulation of carbonates. The Ckg4 horizon is underlying material, with a high degree (g4) of carbonate accumulations.

A moderate humus supply in the first $50 \mathrm{~cm}$ of soil and a low nitrogen level between 0-24 cm was observed. Also, highly acid soil between 43-64 $\mathrm{cm}$, moderately acidic zone between $0-43 \mathrm{~cm}$ and $64-123 \mathrm{~cm}$ and low alkaline zone between depth of 123 and $165 \mathrm{~cm}$ was observed. 
Table 1. Main soil features downstream Bârzava, Caraş-Severin County, Romania

\begin{tabular}{|c|c|c|c|c|c|c|c|}
\hline Horizon & Ap & Ao & $A B$ & Bvg2 & $\mathrm{BCg} 2$ & $\mathrm{Ckg}_{3}$ & $\mathrm{Ckg}_{4}$ \\
\hline Depth (cm) & $0-24$ & -43 & -64 & -96 & -123 & -148 & -165 \\
\hline $\begin{array}{l}\text { Coarse sand }(2.0-0.2 \\
\mathrm{mm})(\%)\end{array}$ & 7.3 & 1.2 & 1.3 & 3.6 & 2.9 & 4.7 & 4.7 \\
\hline $\begin{array}{l}\text { Fine sand } \\
(0.2-0.02 \mathrm{~mm})(\%)\end{array}$ & 60.5 & 60.8 & 58.3 & 53.5 & 59.9 & 69.8 & 73.4 \\
\hline $\begin{array}{l}\text { Dust } \\
(0.02-0.002 \mathrm{~mm})(\%)\end{array}$ & 18.5 & 12.8 & 17.1 & 22.1 & 18.4 & 9.0 & 6.9 \\
\hline $\begin{array}{l}\text { Colloidal clay } \\
(<0.002 \mathrm{~mm})(\%)\end{array}$ & 13.5 & 25.2 & 23.3 & 20.8 & 18.8 & 16.5 & 15.0 \\
\hline $\begin{array}{l}\text { Physical clay } \\
(<0.01 \mathrm{~mm})(\%)\end{array}$ & 28.3 & 33.8 & 31.5 & 26.6 & 24.4 & 17.1 & 17.2 \\
\hline Texture & SM & LN & LL & LL & SF & SM & SM \\
\hline $\begin{array}{l}\text { Apparent density (Da) } \\
\left(\mathrm{g} / \mathrm{cm}^{3}\right)\end{array}$ & 1.4 & 1.5 & 1.6 & 1.6 & & & \\
\hline $\begin{array}{l}\text { Specific density (Ds) } \\
\left(\mathrm{g} / \mathrm{cm}^{3}\right)\end{array}$ & 2.7 & 2.6 & 2.6 & 2.6 & & & \\
\hline Water $\mathrm{pH}$ & 5.3 & 5.2 & 4.9 & 5.7 & 6.2 & 8.2 & 8.4 \\
\hline $\begin{array}{l}\text { Carbonates }\left(\mathrm{CaCO}_{3}\right) \\
(\%)\end{array}$ & & & & & & 5.8 & 5.2 \\
\hline Humus (\%) & 2.4 & 1.4 & 1.1 & & & & \\
\hline Nitrogen index (IN) & 1.9 & & & & & & \\
\hline Humus reserve (t/ha) & 80.3 & 41.6 & 12.0 & 133.9 & & & \\
\hline $\begin{array}{l}\text { Exchange bases (SB) } \\
\text { (me/100) }\end{array}$ & 7.4 & 8.9 & 12.0 & 19.1 & 21.6 & & \\
\hline $\begin{array}{l}\text { Exchange hydrogen } \\
\text { (SH) (me/100) }\end{array}$ & 4.6 & 4.6 & 4.4 & 3.3 & 1.9 & & \\
\hline $\begin{array}{l}\text { Cation exchange } \\
\text { capacity }(\mathrm{T})(\mathrm{me} / 100)\end{array}$ & 11.9 & 13.5 & 16.4 & 22.4 & 85.4 & & \\
\hline $\begin{array}{l}\text { Base saturation } \\
\text { degree }(V)(\%)\end{array}$ & 61.8 & 66.2 & 73.0 & 85.4 & 91.5 & & \\
\hline
\end{tabular}

Legend: Ap-ploughed layer; Ao-ocric horizon; AB- horizon; Bvg2 horizon; BCg2 horizon; Ckg3 horizon; Ckg4 horizon; SM- sandy gray clay; LN - sandy clay; LL- middle clay; SFsandy fine clay;

For soils characterization, agricultural surface with acidic soils distribution from downstream Bârzava River, were systematized from data obtained from administrative-territorial units of the studied area. As far as acid soils are concerned, they cover 47,297 ha of agricultural lands within the studied area (Table 2). These soils share a common feature; low $\mathrm{pH}$ values 
(5.0-6.8), which groups them into two large groups depending on acidification and soil formation type: soils with a B argillic horizon (Bt) and soils with a B cambic horizon (Bv).

Table 2. Distribution of acidis soils in agricultural lands from downstream Bârzava river, Caras-Severin County, Romania

\begin{tabular}{|l|c|c|c|c|}
\hline \multirow{2}{*}{$\begin{array}{l}\text { Commune/ } \\
\text { Town }\end{array}$} & $\begin{array}{l}\text { Total } \\
\text { agricultural } \\
\text { lands (ha) }\end{array}$ & $\begin{array}{c}\text { low } \\
\text { pH: 5.8-6.8 }\end{array}$ & $\begin{array}{c}\text { moderate } \\
\text { pH: 5.1-5.8 }\end{array}$ & $\begin{array}{c}\text { Ongh- } \\
\text { excessive } \\
5.1\end{array}$ \\
\hline Banloc(+Livezile) & 15481 & 6337 & 1832 & --- \\
\hline Denta & 8414 & 4644 & 1712 & --- \\
\hline Deta & 2904 & 1702 & 484 & --- \\
\hline Gătaia (+ Birda) & 19496 & 9850 & 2724 & 83 \\
\hline Ghilad & 10487 & 6023 & 1603 & --- \\
\hline Giera & 8556 & 4479 & 482 & --- \\
\hline Voiteni & 6523 & 3836 & 1506 & --- \\
\hline Total & 71861 & 36871 & 10343 & 83 \\
\hline$\%$ & 66.0 & 51.3 & 14.4 & 0.1 \\
\hline
\end{tabular}

Acidity, expressed as actual acidity $(\mathrm{pH})$ and titratable acidity (exchange and hydrolytic) influences directly (through the root system) the process of nutrition in plants. Acidity influences deeply the process of metabolism by disturbing the formation of protein substances: this is the reason why nitrogen substances remain as amino acids in the root.

Some $\mathrm{pH}$ measurements were achieved, according the literature procedures ${ }^{17,18,19}$ from collected soils and results were presented in Table 3. From measurements we can observed that surface soil is more acidic.

Many studies reported that the soil $\mathrm{pH}$ and heavy metal concentrations are vital factors for plant growth ${ }^{20,21}$. Therefore, in this study the six soil samples were analysed in order to assess the major and trace elements content. The obtained results are presented in the Table 4.

The metal content in collected soil samples was determined using an Inductively Coupled Plasma Mass Spectrometer. Over 50 different metals were identified. Some in higher concentration like $\mathrm{Ca}, \mathrm{Fe}, \mathrm{Mg}, \mathrm{Mn}, \mathrm{Ba}$ some in medium concentration like $\mathrm{Sr}, \mathrm{Na}, \mathrm{Ti}, \mathrm{Zn}, \mathrm{Y}, \mathrm{As}, \mathrm{Co}, \mathrm{Ni}$ and some in low concentration, Sc, Ge, Rb, Zr, Cd. Some rare metals were detected but the concentration was under $0.01 \mathrm{mg} / \mathrm{Kg}$. Some lactinides metals were identified, the highest concentration was $\mathrm{Ce}, \mathrm{Nd}$, La, Gd, Dy. 
Table 3. $\mathrm{pH}$-values for soil samples obtained from Bârzava river, Caras-Severin County, Romania; (A soil sample from Banloc-Livezile pump station area $(0-20 \mathrm{~cm})$;

B. soil sample from Partoş rice plantation area $(0-20 \mathrm{~cm})$; C. soil sample from Voiteni Farm of USAMVBT $(0-20 \mathrm{~cm})$; D. soil sample from wheat plantation area from the Voiteni Farm of USAMVBT $(20-40 \mathrm{~cm})$; E. soil sample from

Banloc- Livezile pump station area $(20-40 \mathrm{~cm})$; F soil sample from Partoş rice plantation area $(20-40 \mathrm{~cm})$ )

\begin{tabular}{|c|l|l|l|l|l|l|}
\hline $\begin{array}{c}\text { Soil } \\
\text { samples }\end{array}$ & A & B & C & D & E & F \\
\hline $\mathrm{pH}$ & 5.3 & 5.6 & 5.4 & 6.1 & 5.9 & 6.1 \\
\hline & & & & & & \\
\hline
\end{tabular}

Table 4. The total metal concentrations in soil samples collected from different areas: A. soil from Banloc-Livezile pump station area $(0-20 \mathrm{~cm})$; B. soil from Partoş rice plantation area $(0-20 \mathrm{~cm})$; C. soil from Voiteni Farm of USAMVBT $(0-20 \mathrm{~cm})$; D. soil from wheat plantation area from Voiteni Farm of USAMVBT

(20-40 cm); E. soil from Banloc-Livezile pump station area $(20-40 \mathrm{~cm})$; F. soil from Partoş rice plantation area $(20-40 \mathrm{~cm})$

\begin{tabular}{|l|c|c|c|c|c|c|}
\hline Element & $\begin{array}{c}\mathrm{A} . \\
\mathrm{C}_{\mathrm{M}}=\mathrm{mg} / \mathrm{kg}\end{array}$ & $\begin{array}{c}\mathrm{B} . \\
\mathrm{C}_{\mathrm{M}}=\mathrm{mg} / \mathrm{kg}\end{array}$ & $\begin{array}{c}\mathrm{C} . \\
\mathrm{C}_{\mathrm{M}}=\mathrm{mg} / \mathrm{kg}\end{array}$ & $\begin{array}{c}\mathrm{D} . \\
\mathrm{C}_{\mathrm{M}}=\mathrm{mg} / \mathrm{kg}\end{array}$ & $\begin{array}{c}\mathrm{E} . \\
\mathrm{C}_{\mathrm{M}}=\mathrm{mg} / \mathrm{kg}\end{array}$ & $\begin{array}{c}\mathrm{F} . \\
\mathrm{C}_{\mathrm{M}}=\mathrm{mg} / \mathrm{kg}\end{array}$ \\
\hline $\mathbf{L i}$ & 1.63 & 0.88 & 2.07 & 1.95 & 0.97 & 1.19 \\
\hline $\mathbf{B e}$ & 0.61 & 0.48 & 0.72 & 0.73 & 0.28 & 0.55 \\
\hline $\mathbf{N a}$ & 29.65 & 75.91 & 54.87 & 70.39 & 15.72 & 71.89 \\
\hline $\mathbf{M g}$ & 3474.8 & 1796.4 & 2627.9 & 2487.2 & 1503.8 & 1793.6 \\
\hline $\mathbf{C a}$ & 8846.2 & 4338.5 & 6946.7 & 7063.1 & 3369.4 & 4843.1 \\
\hline $\mathbf{S c}$ & 0.64 & 0.94 & 0.65 & 0.62 & 0.30 & 0.98 \\
\hline $\mathbf{T i}$ & 14.76 & 8.23 & 12.69 & 12.93 & 6.15 & 9.23 \\
\hline $\mathbf{V}$ & 23.38 & 19.32 & 15.96 & 14.16 & 10.29 & 21.07 \\
\hline $\mathbf{C r}$ & 4.17 & 2.51 & 4.12 & 3.53 & 2.15 & 3.22 \\
\hline $\mathbf{M n}$ & 307.03 & 112.57 & 477.34 & 379.92 & 128.59 & 132.31 \\
\hline $\mathbf{F e}$ & 320.78 & 3635.8 & 2560.7 & 2211.4 & 1363.6 & 283.87 \\
\hline $\mathbf{C o}$ & 5.25 & 6.91 & 6.36 & 6.27 & 2.11 & 3.41 \\
\hline $\mathbf{N i}$ & 7.69 & 4.61 & 9.22 & 8.50 & 3.44 & 5.37 \\
\hline $\mathbf{C u}$ & 8.45 & 7.68 & 7.75 & 6.56 & 3.30 & 8.09 \\
\hline $\mathbf{Z n}$ & 14.76 & 17.69 & 15.12 & 15.60 & 6.02 & 20.97 \\
\hline $\mathbf{G a}$ & 0.95 & 0.59 & 0.88 & 0.76 & 0.42 & 0.69 \\
\hline $\mathbf{G e}$ & 0.03 & 0.02 & 0.03 & 0.02 & 0.01 & 0.02 \\
\hline $\mathbf{A s}$ & 7.32 & 4.72 & 5.22 & 4.99 & 2.74 & 4.90 \\
\hline $\mathbf{S e}$ & $<0.01$ & 0.15 & $<0.01$ & $<0.01$ & 0.09 & $<0.01$ \\
\hline $\mathbf{R b}$ & 0.43 & 0.25 & 0.43 & 0.47 & 0.16 & 0.25 \\
\hline $\mathbf{S r}$ & 24.17 & 16.32 & 20.61 & 19.51 & 9.48 & 18.45 \\
\hline $\mathbf{Y}$ & 9.12 & 7.16 & 10.11 & 9.79 & 3.75 & 8.25 \\
\hline $\mathbf{Z r}$ & 0.39 & 0.15 & 0.25 & 0.21 & 0.12 & 0.18 \\
\hline & & & & & & \\
\hline
\end{tabular}




\begin{tabular}{|c|c|c|c|c|c|c|}
\hline Element & $\begin{array}{c}\text { A. } \\
\mathrm{C}_{\mathrm{M}}=\mathrm{mg} / \mathrm{kg}\end{array}$ & $\begin{array}{c}\mathrm{B} . \\
\mathrm{C}_{\mathrm{M}}=\mathrm{mg} / \mathrm{kg} \\
\end{array}$ & $\begin{array}{c}\mathrm{C} . \\
\mathrm{C}_{\mathrm{M}}=\mathrm{mg} / \mathrm{kg}\end{array}$ & $\begin{array}{c}\mathrm{D} . \\
\mathrm{C}_{\mathrm{M}}=\mathrm{mg} / \mathrm{kg}\end{array}$ & $\begin{array}{c}\mathrm{E} . \\
\mathrm{C}_{\mathrm{M}}=\mathrm{mg} / \mathrm{kg}\end{array}$ & $\begin{array}{c}\mathrm{F} . \\
\mathrm{C}_{\mathrm{M}}=\mathrm{mg} / \mathrm{kg}\end{array}$ \\
\hline $\mathrm{Nb}$ & 0.03 & 0.02 & 0.02 & 0.02 & 0.01 & 0.02 \\
\hline Mo & 0.03 & 0.01 & 0.01 & $<0.01$ & $<0.01$ & $<0.01$ \\
\hline $\mathbf{R u}$ & $<0.01$ & $<0.01$ & $<0.01$ & $<0.01$ & $<0.01$ & $<0.01$ \\
\hline $\mathbf{R h}$ & $<0.01$ & $<0.01$ & $<0.01$ & $<0.01$ & $<0.01$ & $<0.01$ \\
\hline $\mathbf{P d}$ & $<0.01$ & $<0.01$ & $<0.01$ & $<0.01$ & $<0.01$ & $<0.01$ \\
\hline $\mathbf{A g}$ & 0.06 & 0.06 & 0.04 & 0.04 & 0.02 & 0.06 \\
\hline $\mathbf{C d}$ & 0.14 & 0.20 & 0.13 & 0.11 & 0.05 & 0.22 \\
\hline $\ln$ & $<0.01$ & 0.03 & $<0.01$ & $<0.01$ & $<0.01$ & 0.03 \\
\hline Sn & 0.04 & 0.04 & 0.02 & 0.02 & $<0.01$ & 0.03 \\
\hline $\mathbf{S b}$ & 0.02 & $<0.01$ & $<0.01$ & 0.01 & $<0.01$ & $<0.01$ \\
\hline Te & $<0.01$ & $<0.01$ & $<0.01$ & $<0.01$ & $<0.01$ & $<0.01$ \\
\hline I & 0.01 & $<0.01$ & 0.04 & 0.04 & $<0.01$ & $<0.01$ \\
\hline Cs & $<0.01$ & $<0.01$ & $<0.01$ & $<0.01$ & $<0.01$ & $<0.01$ \\
\hline $\mathrm{Ba}$ & 127.69 & 76.31 & 140.36 & 131.24 & 46.41 & 93.10 \\
\hline La & 10.01 & 8.11 & 10.89 & 10.07 & 3.68 & 9.53 \\
\hline $\mathrm{Ce}$ & 24.97 & 19.13 & 27.38 & 24.63 & 9.13 & 22.79 \\
\hline $\mathrm{Pr}$ & 3.02 & 2.29 & 3.29 & 3.09 & 1.10 & 2.78 \\
\hline Nd & 12.95 & 10.02 & 14.34 & 13.61 & 1.56 & 12.06 \\
\hline Sm & 1.09 & 0.81 & 1.24 & 1.19 & 0.36 & 1.01 \\
\hline Eu & 0.28 & 0.21 & 0.31 & 0.29 & 0.09 & 0.26 \\
\hline Gd & 6.76 & 1.04 & 7.47 & 7.07 & 0.41 & 6.05 \\
\hline Tb & 1.02 & 0.14 & 1.13 & 1.08 & 0.06 & 0.17 \\
\hline Dy & 6.53 & 0.78 & 7.05 & 6.86 & 0.33 & 0.97 \\
\hline $\mathrm{Ho}$ & 1.68 & 0.14 & 1.82 & 1.76 & 0.06 & 0.18 \\
\hline $\mathrm{Er}$ & 0.53 & 0.40 & 0.56 & 0.55 & 0.17 & 0.49 \\
\hline Tm & 0.07 & 0.06 & 0.08 & 0.07 & 0.02 & 0.07 \\
\hline $\mathbf{Y b}$ & 0.50 & 0.39 & 0.52 & 0.50 & 0.16 & 0.47 \\
\hline Lu & 0.08 & 0.06 & 0.08 & 0.08 & 0.02 & 0.07 \\
\hline $\mathrm{Hf}$ & 0.01 & 0.01 & 0.01 & 0.01 & $<0.01$ & 0.01 \\
\hline Ta & $<0.01$ & $<0.01$ & $<0.01$ & $<0.01$ & $<0.01$ & $<0.01$ \\
\hline $\mathbf{W}$ & $<0.01$ & $<0.01$ & $<0.01$ & $<0.01$ & $<0.01$ & $<0.01$ \\
\hline $\mathbf{R e}$ & $<0.01$ & $<0.01$ & $<0.01$ & $<0.01$ & $<0.01$ & $<0.01$ \\
\hline Os & $<0.01$ & $<0.01$ & $<0.01$ & $<0.01$ & $<0.01$ & $<0.01$ \\
\hline Ir & $<0.01$ & $<0.01$ & $<0.01$ & $<0.01$ & $<0.01$ & $<0.01$ \\
\hline $\mathrm{Pt}$ & $<0.01$ & $<0.01$ & $<0.01$ & $<0.01$ & $<0.01$ & $<0.01$ \\
\hline $\mathbf{A u}$ & $<0.01$ & $<0.01$ & $<0.01$ & $<0.01$ & $<0.01$ & $<0.01$ \\
\hline $\mathrm{Hg}$ & $<0.01$ & 0.01 & 0.01 & $<0.01$ & $<0.01$ & 0.02 \\
\hline TI & $<0.01$ & $<0.01$ & $<0.01$ & $<0.01$ & $<0.01$ & $<0.01$ \\
\hline $\mathrm{Pb}$ & $<0.01$ & $<0.01$ & $<0.01$ & $<0.01$ & $<0.01$ & $<0.01$ \\
\hline $\mathbf{B i}$ & 0.15 & 0.42 & 0.09 & 0.09 & 0.05 & $<0.01$ \\
\hline
\end{tabular}




\section{CONCLUSIONS}

Soil from downstream Bârzava River was characterized and main features were presented. The six soil collected samples were characterized and analysed and in order to assess the major and trace elements content and to determine the acidity of soils. The $\mathrm{pH}$ of soils was determined from different depths, the surface soil being more acidic. It was revealed that $\mathrm{Ca}$, $\mathrm{Fe}, \mathrm{Mg}, \mathrm{Mn}, \mathrm{Ba}$ were the most abundant elements in the studied soil samples. It is known that the $\mathrm{pH}$ and heavy metal concentrations are vital factors for plant growth and the obtained results could be use for elaboration of some soil bioremediation techniques.

\section{EXPERIMENTAL SECTION}

\section{Material and methods}

Six soil samples were collected from different areas, from lands downstream Bârzava River, Caraş-Severin County, Romania: a soil sample from Banloc-Livezile pump station area $(0-20 \mathrm{~cm})$; B. soil sample from Partoş rice plantation area $(0-20 \mathrm{~cm})$; C. soil sample from Voiteni Farm of USAMVBT $(0-20 \mathrm{~cm})$; D. soil sample from wheat plantation area from Voiteni Farm of USAMVBT $(20-40 \mathrm{~cm})$; E. soil sample from Banloc-Livezile pump station area $(20-40 \mathrm{~cm})$; $\mathrm{F}$ soil sample from Partoş rice plantation area $(20-40 \mathrm{~cm})$.

The metal contents were determined by Inductively Coupled Plasma Mass Spectrometer (SCIEX Perkin Elmer Elan DRC II) using a semiquantitative analysis. Merck ICP multi-element standard solution VI was diluted and used for factor response calibration, while the dynamic reaction cell was used in rf-only mode (no gas). Oxides and double charged ions were kept below 3\%, plasma power was set at $1450 \mathrm{~W}$ and the instrument was optimised for lowest signal/noise raise before measurements. Germany).

All reactive and standards were purchased from Merck (Darmstadt,

\section{Sample preparation and $\mathrm{pH}$ measurement}

The soil samples were air-dried and grounded to $2 \mathrm{~mm}$. Soil samples $\mathrm{pH}$ were measured in soil-water slurry $(1: 5, \mathrm{w} / \mathrm{v})$ with a $\mathrm{pH}$ meter. The $\mathrm{pH}$ meter was calibrate according to manufacturer's instructions using buffer solutions $(\mathrm{pH} 4.0$ - 10.0). The $\mathrm{pH}$ measurement was performed at room temperature $\left(20^{\circ}-23^{\circ} \mathrm{C}\right)$. 


\section{Extraction and determination of metals from soil samples}

The total metal content of the soil samples were determined on centrifugated extracts $(0.5 \mathrm{~g} / \mathrm{mL})$ obtained from $5 \mathrm{~g}$ samples which were digested with $10 \mathrm{ml} 1 \mathrm{M} \mathrm{HCl}$. The total metal determinations were conducted by Inductively Coupled Plasma Mass Spectrometer (SCIEX Perkine Elmer Elan DRC II).

\section{ACKNOWLEDGMENTS}

The authors thank for financial support from the National Authority for Scientific Research and Innovation (ANCSI) Core Program (Project No. 16.40.02.01).

\section{REFERENCES}

1. H.R. Uexküll, E. Mutert, Plant and Soil, 1995, 171, 1-15.

2. J.H. Guo, X.J. Liu, Y. Zhang, J.L Shen, W.X., Han, W.F. Zhang, P. Christie, K.W.T. Goulding, P.M. Vitousek, F. S. Zhang, Science, 2010, 327, 1008-1010.

3. J. Polomski, N. Kuhn, "Root Research Methods. In: Waisel, Y., Eshel, A., Kafkafi U. and Dekker, M., Eds., Plant Roots: The Hidden Half" (third), New York, 2002, 313-314.

4. V.A. Vitorello, F.R. Capaldi, V.A. Stefanuto, Brazilian Journal of Plant Physiology, 2005, 17, 129-143.

5. S.M. Alam, S.S.M. Naqvi, R. Ansari, "Impact of Soil pH on Nutrient Uptake by Crop Plants. In: Pessarakli, M.", Ed., Handbook of Plant and Crop Stress, New York, 1999, 51-60.

6. J.F. Ma, International Review of Cytology, 2007, 264, 225-252.

7. A. Robson, Soil Acidity and Plant Growth, $1^{\text {st } E d ., ~ A c a d e m i c ~ P r e s s, ~ 1989, ~} 318$.

8. W.Y. Shi, H.B. Shao, M. Shao, S. Du, Journal of Hazardous Materials, 2009, $170(1), 1-6$.

9. F.R. Troeh, L.M. Thomson, "Soils and soil fertility", Ed. Blackwell, 2005, 489.

10. D. Tărău, Gh. rogobete, A. Grozav, D. Dicu, A. Tărău, Research Journal of Agricultural Science, 2012, 44 (3), 293-298.

11. Gh. Rogobete, D. Ţărău, "Soils and their improvement", Ed. Marineasa, 1997, Timisoara.

12. M. Dumitru, C. Ciobanu, R. Lacatusu, L. Latis, G. Gament, M. Dracea, St. Carstea, E. Dulvara, D. Plaxienco, B. Kovacsovics, R. Enache, M.D. Motelica, M. Alexandrina, N. Vranceanu "Protectia Mediului in Agricultura", Ed. Helicon, 2000, p.16-42. 
13. a) I. Niţu, C. Răuţă, M. Drăcea, Agropedoameliorative works, vol I, Ceres Publishing House, 1988, Bucharest; b) I. Niţu, C. Răuţă, M. Drăcea, Agropedoameliorative works, vol II, Ceres Publishing House, 1990, Bucharest.

14. I. Borza, D. Tărău, R. Jarabă, I. Tărău, "The impact of economical and social ctivities on soil quality and use of agricultural lands in the peri-urban area URUIOC STELA et al.: Evaluation od the degree of pollution with heavy metals of soils of Timisoara town", vol. XXXVII, Ed. Agroprint, Timişoara, 2005, ISSN 1221-5279, 258-263.

15. N. Sarwar, Saifullah, S.S. Malhi, M.H. Zia, A. Naeem, S. Bibi, G. Farid, Journal of the Science of Food and Agriculture, 2010, 90, 925.

16. A. Mahmood, R. Naseem Malik, Arabian Journal of Chemistry, 2014, 7, 91.

17. A. Behnood, K. Van Tittelboom, N. De Belie, Construction and Building Materials, 2016, 105, 176.

18. R.O. Miller, D.E. Kissel, Soil Science Society of America Journal, 2008, 74, 310.

19. M. Hansen, T. Bang-Andreasen, H. Sorensen, M. Ingerslev, Forest Ecology and Management, 2017, 406, 274.

20. M. Vítkov, S. Rakosov, Z. Michalkov, M. Komarek, Journal of Environmental Management, 2017, 186, 268.

21. S. Willscher, L. Jablonski, Z. Fona, R. Rahmi, J.Wittig, Hydrometallurgy, 2017, 168, 153. 